\title{
Paciente cardiopata em tratamento de câncer: Implicações para o cuidado de enfermagem
}

\author{
Cardiac patient undergoing cancer treatment: Implications for nursing care \\ Paciente cardíaco en tratamiento oncológico: Implicaciones para los cuidados de enfermería
}

Recebido: 22/04/2021 | Revisado: 01/05/2021 | Aceito: 14/05/2021 | Publicado: 22/05/2021

\author{
Victor Hugo de Paula Flauzino \\ ORCID: https://orcid.org/0000-0001-5156-0030 \\ Faculdade Unyleya, Brasil \\ E-mail: prof.victorflauzino@unyleya.edu.br \\ Priscila Gramata da Silva Vitorino \\ ORCID: https://orcid.org/0000-0002-1201-6945 \\ Associação Brasileira de Enfermeiros Cientistas, Brasil \\ E-mail: prigramaenf@yahoo.com.br \\ Luana de Oliveira Hernandes \\ ORCID: http://orcid.org/0000-0001-8828-7146 \\ Associação Brasileira de Enfermeiros Cientistas, Brasil \\ E-mail: luanaoliveira2306@gmail.com \\ Daiana Moreira Gomes \\ ORCID: https://orcid.org/0000-0001-9387-0619 \\ Associação Brasileira de Enfermeiros Cientistas, Brasil \\ E-mail: daigomes_87@hotmail.com \\ Jonas Magno dos Santos Cesário \\ ORCID: https://orcid.org/0000-0003-1785-3555 \\ Faculdade Unyleya, Brasil \\ E-mail: prof.jonasmagno@unyleya.edu.br
}

\begin{abstract}
Resumo
Pessoas que necessitam de tratamento oncológico podem desenvolver doenças cardiovasculares devido ao tratamento oncológico utilizado, como por exemplo, a quimioterapia e a radioterapia. Esses tipos de tratamentos, podem causar cardiotoxicidade, a qual, é gerada por meio de do acúmulo de certas substâncias químicas no coração. Este estudo buscou descrever as particularidades do tratamento contra o câncer em pacientes cardiopatas, além de descrever os cuidados de enfermagem no paciente com cardiopatia que está em tratamento de câncer com uso de quimioterapia e radioterapia. A pesquisa é uma revisão bibliográfica de abordagem descritiva e qualitativa. Faz-se necessário a elaboração e implantação de diretrizes para o uso de quimioterapia e radioterapia, pois somente com uma prática clínica padronizada e sistematizada, será possível garantir a segurança no tratamento do paciente oncológico com cardiopatia. Os principais cuidados de enfermagem em pacientes oncológicos com cardiopatia são: intervenções de educação de autocuidado, monitoramento de sinais vitais, mudanças de decúbito de acordo com a necessidade previamente diagnosticada pelo enfermeiro, posicionamento do paciente, gerenciamento de medicamentos, programas de tratamento como: soroterapia, preparo do cólon, cuidados com tubo de infusão, registros médicos, de enfermagem e referências de exames, controle da ingesta hídrica, garantia da permeabilidade das vias aéreas, curativo diário e controle da dor. Espera-se que este trabalho contribua para melhorar a sistematização da assistência ao paciente oncológico cardiopata, assim como contribuir com publicações futuras a respeito dessa temática.
\end{abstract}

Palavras-chave: Cardiopatia; Oncologia; Cuidados de enfermagem.

\begin{abstract}
People who need cancer treatment can develop cardiovascular diseases due to the cancer treatment used, such as chemotherapy and radiotherapy. These types of treatments can cause cardiotoxicity, which is generated by the accumulation of certain chemicals in the heart. This study sought to describe the particularities of cancer treatment in cardiac patients, in addition to describing nursing care for patients with heart disease who are undergoing cancer treatment using chemotherapy and radiotherapy. The research is a bibliographic review with a descriptive and qualitative approach. It is necessary to develop and implement guidelines for the use of chemotherapy and radiotherapy, as only with a standardized and systematized clinical practice, it will be possible to guarantee safety in the treatment of cancer patients with heart disease. The main nursing care in cancer patients with heart disease are self-care education interventions, monitoring vital signs, decubitus changes according to the need previously diagnosed by the nurse, patient positioning, medication management, treatment programs such as: serotherapy, colon preparation, infusion tube care, medical, nursing records and exam references, control of fluid intake, guarantee of
\end{abstract}


airway permeability, daily dressing and pain control. It is hoped that this work will contribute to improving the systematization of care for cardiac cancer patients, as well as contributing to future publications on this topic.

Keywords: Heart disease; Oncology; Nursing care.

\begin{abstract}
Resumen
Las personas que necesitan tratamiento contra el cáncer pueden desarrollar enfermedades cardiovasculares debido al tratamiento contra el cáncer utilizado, como la quimioterapia y la radioterapia. Este tipo de tratamientos pueden provocar cardiotoxicidad, que se genera por la acumulación de determinadas sustancias químicas en el corazón. Este estudio buscó describir las particularidades del tratamiento del cáncer en pacientes con enfermedades cardíacas, además de describir los cuidados de enfermería para pacientes con enfermedades cardíacas que están en tratamiento contra el cáncer con quimioterapia y radioterapia. La investigación es una revisión bibliográfica con enfoque descriptivo y cualitativo. Es necesario desarrollar e implementar pautas para el uso de quimioterapia y radioterapia, porque solo con una práctica clínica estandarizada y sistematizada, será posible garantizar la seguridad en el tratamiento de pacientes oncológicos con cardiopatías. Los principales cuidados de enfermería en pacientes oncológicos con cardiopatías son: intervenciones de educación en el autocuidado, seguimiento de signos vitales, cambios de decúbito según la necesidad previamente diagnosticada por la enfermera, posicionamiento del paciente, manejo de medicamentos, programas de tratamiento como: seroterapia, preparación de colon, cuidado del tubo de infusión, registros médicos, de enfermería y referencias de exámenes, control de la ingesta de líquidos, garantía de permeabilidad de la vía aérea, vendaje diario y control del dolor. Se espera que este trabajo contribuya a mejorar la sistematización de la atención al paciente con cáncer cardíaco, además de contribuir a futuras publicaciones sobre este tema.
\end{abstract}

Palabras clave: Enfermedad cardíaca; Oncología; Cuidado de enfermera.

\title{
1. Introdução
}

As duas principais causas de morte no mundo estão associadas a problemas cardiovasculares e ao câncer. Com a área da saúde em constante evolução tecnológica, novos tratamentos foram testados e experimentados, o que resultou no aumento das chances de vida dos pacientes acometidos por essas morbidades. Porém, por mais que essa evolução seja uma realidade, ainda é alto o número de pacientes acometidos por essas doenças e o número de mortes continua a subir.

No Brasil, devido às grandes mudanças, a expectativa de vida aumenta a cada dia mais, e, por conta disso, aumenta-se a população idosa do país. Sabe-se que as doenças cardiovasculares e o câncer são doenças crônicas e estão, na maioria das vezes, associadas à idade e ao estilo de vida da pessoa. Portanto, com a população mais idosa, maiores são as chances do desenvolvimento de doenças crônicas como o câncer, o que leva a aumento nos índices de procura por tratamentos com antineoplásicos (Santos, Siqueira \& Renni, 2019).

Estudiosos explicam que pessoas que necessitam de tratamento oncológico podem desenvolver doenças cardiovasculares devido ao tratamento oncológico utilizado, como por exemplo, a quimioterapia e a radioterapia. Esses tipos de tratamentos, podem causar cardiotoxicidade, a qual, é gerada por meio do acúmulo de certas substâncias químicas no coração. Esse acúmulo, resultante de substâncias presentes nos antineoplásicos, resulta em problemas cardíacos graves ou agrava problemas cardíacos já existentes. As complicações cardiovasculares desenvolvidas ou agravadas por meio do tratamento oncológico, se torna então uma vilã, que pode causar maior número de morte do que o próprio câncer (Hajjar et al., 2020).

O paciente em tratamento oncológico com risco de desenvolvimento de afecções cardíacas ou de agravar as já existentes requerem um planejamento de cuidado multidisciplinar, voltado para a qualidade de vida e prevenção da doença. A identificação dos diagnósticos, acoplada ao histórico do paciente, é essencial para que se estude as reais necessidades desse paciente e se trace uma estratégia de cuidados, com o objetivo de melhorar o cuidado e consequente prevenção e tratamento da doença (Mesquita, 2016).

Ao partir deste ponto, sabe-se que cabe ao enfermeiro a responsabilidade por acompanhar e prestar os principais cuidados ao paciente durante o seu processo de tratamento, ao se basear no cuidado que atenda às principais demandas do 
paciente de forma integral. Os cuidados prestados aos pacientes oncológicos, requerem que equipe a enfermagem faça uma abordagem ao paciente, sem esquecer da família, com o intuito de proporcionar uma assistência humanizada e direcionada ao paciente integralmente. Dessa forma, elaborou-se esse estudo com o objetivo de descrever as particularidades do tratamento contra o câncer em pacientes cardiopatas, além de descrever os cuidados de enfermagem no paciente com cardiopatia que está em tratamento de câncer com uso de quimioterapia e radioterapia.

\section{Metodologia}

O presente estudo é uma revisão bibliográfica com uma abordagem descritiva e qualitativa. Conforme Cesário, Flauzino e Mejia (2020), a revisão bibliográfica é fundamentada em literatura na qual foi construída por meio de artigos científicos e que foram publicados nas revistas cientificas. Buscou-se reunir evidências para responder a seguinte pergunta de pesquisa: como o tratamento contra o câncer em pacientes cardiopatas afeta os cuidados de enfermagem?

No DeCS (Descritores em Ciências da Saúde), encontrou-se os seguintes descritores: Cardiopatia; Oncologia; Cuidados de enfermagem. Os bancos de dados utilizados foram na SciELO (Scientific Electronic Library Online) Google Acadêmico e BVS (biblioteca virtual em saúde). No Google Acadêmico utilizou-se cada um dos descritores entre aspas ("'). Na BVS (biblioteca virtual em saúde), foi utilizado a opção pesquisa avançada, selecionada as bases da BDENF (Banco de Dados em Enfermagem), LILACS (Literatura Latino-Americana e do Caribe em Ciências da Saúde) e o operador lógico booleano "OR" e "AND". Na SciELO, foi utilizada a opção pesquisa avançada e o operador lógico booleano "OR" e "AND".

Estabeleceu-se como critérios de inclusão, artigos acadêmicos publicados entre 2013 e 2021, na língua portuguesa, disponíveis de forma gratuita e nos bancos de dados já mencionados, que respondessem à pergunta de pesquisa. Excluíram-se artigos repetidos encontrados nas bases de dados, resumos, artigos inferiores a 2013 e artigos que não respondiam o problema da pesquisa. A coleta dos dados foi realizada no mês de março, por 5 pesquisadores de forma independente. Os resultados das buscas pelos dados e do número final de publicações que irão compor a revisão serão apresentados por meio do fluxograma de Prisma, elaborado por Peters et al., 2015. A utilização do fluxograma contribuiu para uma melhor organização da busca por estudos e para melhorar a consistência do relato da revisão sistemática. O fluxograma mostra como foi organizada as pesquisas realizadas nos Bancos de dados da BVS, ScIELO e Google escolar, na qual foi organizada em 4 etapas distintas compostas pelos critérios de exclusão e inclusão utilizados durante a pesquisa, conforme pode ser observado na Figura 1. 
Figura 1: Fluxograma de Prisma.

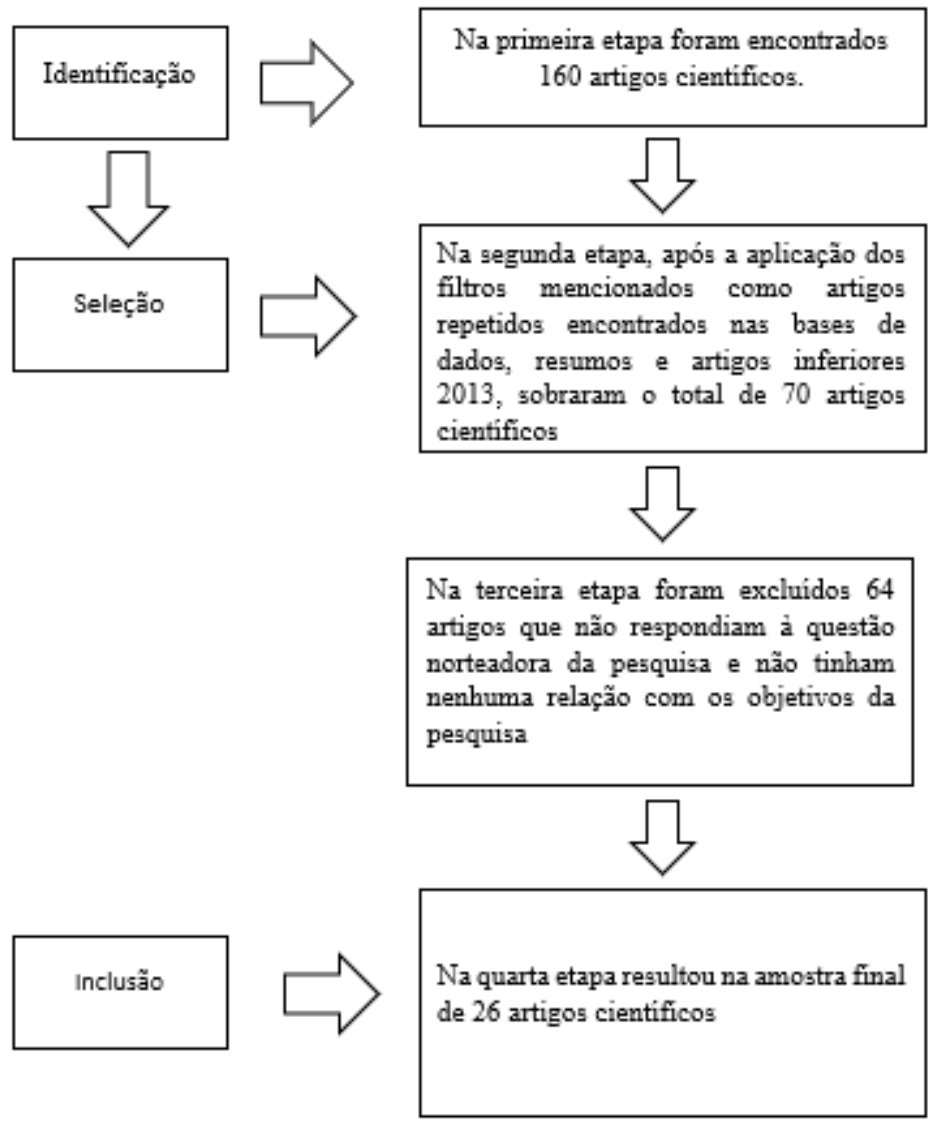

Fonte: Autores (2021).

\section{Resultados}

A tabela 1 foi desenvolvida para visualizar a distribuição inicial dos artigos científicos que foram encontrados durante a busca por periódicos nas bases de dados da ScIELO, Google Acadêmico e BVS.

Tabela 1 - Artigos inclusos neste estudo.

\begin{tabular}{c|c|c}
\hline \multirow{2}{*}{ Base de dados } & \multicolumn{2}{|c}{ Artigos } \\
\cline { 2 - 3 } & Total & Incluídos \\
\hline ScIELO & 35 & 5 \\
\hline BVS & 55 & 10 \\
\hline Google Acadêmico & 70 & 11 \\
\hline
\end{tabular}

Fonte: Autores (2021).

Os resultados encontrados durante a elaboração da pesquisa estão divididos em duas categorias que estão apresentadas pelas letras A e B, a categoria A apresenta as particularidades do tratamento contra o câncer em pacientes cardiopatas. na categoria B mostra os cuidados de enfermagem no paciente com cardiopatia que está em tratamento de câncer com uso de quimioterapia e radioterapia. No Quadro 1, apresentado a seguir, foram organizados os artigos que foram utilizados no desenvolvimento da revisão da literatura, com as seguintes variáveis: autor/ano, título, objetivo e tipo de estudo. 
Quadro 1. Artigos incluídos na categoria temática A.

\begin{tabular}{|c|c|c|c|}
\hline Autor/ano & Título & Objetivos & Tipo de estudo \\
\hline Fiuza et al., 2016 & $\begin{array}{l}\text { Organização e implementação de } \\
\text { uma consulta de cardio-oncologia. }\end{array}$ & $\begin{array}{l}\text { Realizar a revisão do estado da arte e } \\
\text { recomendar práticas de atuação clínica. }\end{array}$ & Estado da arte \\
\hline Mesquita, 2016 & O Surgimento da Cardio-Oncologia & Discutir o surgimento da cardio-oncologia & Revisão integrativa \\
\hline $\begin{array}{l}\text { Mori, Bustamante \& } \\
\text { Bustamante, } 2016\end{array}$ & $\begin{array}{l}\text { Insuficiência cardíaca por } \\
\text { quimioterapia. }\end{array}$ & $\begin{array}{l}\text { Relatar a frequência de efeitos colaterais do } \\
\text { nível cardíaco (cardiotoxicidade) causados } \\
\text { pela quimioterapia. }\end{array}$ & $\begin{array}{l}\text { Estudo } \\
\text { experimental. }\end{array}$ \\
\hline $\begin{array}{l}\text { Acosta-Garduño \& } \quad \& \\
\text { Guillen-Rojas, } 2017\end{array}$ & $\begin{array}{l}\text { Riesgo cardiovascular en cirugía } \\
\text { oncológica. }\end{array}$ & $\begin{array}{l}\text { A redução de complicações cardiovasculares } \\
\text { durante ou após } \\
\text { Cirurgia oncológica }\end{array}$ & $\begin{array}{l}\text { Revisão } \\
\text { literatura }\end{array}$ \\
\hline Fonseca, 2017 & $\begin{array}{l}\text { O tratamento oncológico e a doença } \\
\text { arterial coronariana. }\end{array}$ & $\begin{array}{l}\text { Assistência especializada é retornar o paciente } \\
\text { ao tratamento o mais precocemente possível e, } \\
\text { na fase tardia, evitar a morte por doença } \\
\text { cardiovascular. }\end{array}$ & $\begin{array}{l}\text { Revisão } \\
\text { literária. }\end{array}$ \\
\hline $\begin{array}{l}\text { Santos \& Gallafrio, } \\
2017\end{array}$ & $\begin{array}{l}\text { Cardio-oncologia na população } \\
\text { pediátrica }\end{array}$ & $\begin{array}{l}\text { Analisar os principais efeitos colaterais } \\
\text { cardiovasculares no tratamento do câncer em } \\
\text { pacientes pediátricos. }\end{array}$ & $\begin{array}{l}\text { Estudo de } \\
\text { rastreamento }\end{array}$ \\
\hline Borges et al., 2018 & $\begin{array}{l}\text { Fadiga: Um Sintoma Complexo e } \\
\text { seu Impacto no Câncer e na } \\
\text { Insuficiência Cardíaca. }\end{array}$ & $\begin{array}{l}\text { Discutir os novos conceitos na avaliação da } \\
\text { fadiga em pacientes oncológicos. }\end{array}$ & Artigo de revisão. \\
\hline Abelin \& Renni, 2019 & $\begin{array}{l}\text { Insuficiência Cardíaca no Paciente } \\
\text { Oncológico: Preditores de Risco. }\end{array}$ & Preditores de risco em pacientes oncológicos. & Artigo de opinião. \\
\hline Barros et al., 2019 & $\begin{array}{l}\text { Alteração Contrátil Segmentar } \\
\text { Ventricular Esquerda é Preditor } \\
\text { Independente de Cardiotoxicidade } \\
\text { em Pacientes com Câncer de Mama } \\
\text { em Tratamento Quimioterápico. }\end{array}$ & $\begin{array}{l}\text { Analisar a associação entre o surgimento de } \\
\text { ACSVE e o desenvolvimento de } \\
\text { cardiotoxicidade em pacientes com câncer de } \\
\text { mama em tratamento com quimioterapia. }\end{array}$ & Estudo de Coorte \\
\hline Costa et al., 2019 & $\begin{array}{l}\text { Quimioterápico promove inflamação } \\
\text { no tecido cardíaco. }\end{array}$ & $\begin{array}{l}\text { Analisou parâmetros inflamatórios no coração } \\
\text { de ratos Wistar tratados com 5-FU em doses } \\
\text { semelhantes às usadas na quimioterapia em } \\
\text { humanos. }\end{array}$ & $\begin{array}{l}\text { Pesquisa } \\
\text { experimental }\end{array}$ \\
\hline $\begin{array}{l}\text { Oliveira \& Malachias, } \\
2019\end{array}$ & $\begin{array}{l}\text { Novos horizontes da Cardio- } \\
\text { Oncologia }\end{array}$ & $\begin{array}{l}\text { Descrever a importância da Cardio-Oncologia } \\
\text { no tratamento das complicações de pacientes } \\
\text { oncológicos em uso de quimioterapia. }\end{array}$ & $\begin{array}{l}\text { Revisão } \\
\text { literatura }\end{array}$ \\
\hline
\end{tabular}




\begin{tabular}{|c|c|c|c|}
\hline Hajjar et al., 2020 & $\begin{array}{l}\text { Diretriz Brasileira de Cardio- } \\
\text { oncologia }-2020\end{array}$ & $\begin{array}{l}\text { Renovar o conhecimento e promover a } \\
\text { implementação da abordagem racional e } \\
\text { sistemática das complicações cardiovasculares } \\
\text { no paciente oncológico }\end{array}$ & $\begin{array}{l}\text { Revisão } \\
\text { Literatura }\end{array}$ \\
\hline Macêdo et al., 2020 & $\begin{array}{l}\text { Fatores associados ao excesso de } \\
\text { adiposidade em pacientes com } \\
\text { câncer de mama sob tratamento } \\
\text { quimioterápico em um hospital } \\
\text { oncológico de referência em } \\
\text { Pernambuco - Brasil. }\end{array}$ & $\begin{array}{l}\text { Comparar a composição corpórea em } \\
\text { pacientes com diagnóstico de câncer de mama } \\
\text { em tratamento clínico atendidas no Setor de } \\
\text { Quimioterapia do Hospital do Câncer de } \\
\text { Pernambuco. }\end{array}$ & Estudo transversal. \\
\hline $\begin{array}{l}\text { Pinna Junior et al., } \\
2020\end{array}$ & $\begin{array}{l}\text { O uso de dispositivos cardíacos } \\
\text { eletrônicos implantáveis durante } \\
\text { tratamento oncológico: uma revisão }\end{array}$ & $\begin{array}{l}\text { Relatar o uso de dispositivos cardíacos } \\
\text { eletrônicos implantáveis durante o tratamento } \\
\text { oncológico. }\end{array}$ & $\begin{array}{l}\text { Revisão } \\
\text { literatura }\end{array}$ \\
\hline
\end{tabular}

Fonte: Autores (2021).

No Quadro 2, são apresentados os artigos utilizados para desenvolver a categoria temática B, com as seguintes variáveis: autor/ano, título, objetivo e tipo de estudo.

Quadro 2. Artigos incluídos na categoria temática B.

\begin{tabular}{|c|c|c|c|}
\hline Autor/ano & Título & Objetivos & Tipo de estudo \\
\hline Silva et al., 2013 & $\begin{array}{l}\text { Cuidados de enfermagem à criança com } \\
\text { câncer: uma revisão integrativa da } \\
\text { literatura }\end{array}$ & $\begin{array}{l}\text { Revisar a produção científica acerca dos } \\
\text { cuidados de enfermagem à criança com câncer. }\end{array}$ & $\begin{array}{l}\text { Revisão integrativa da } \\
\text { literatura. }\end{array}$ \\
\hline $\begin{array}{l}\text { Vieira, } \quad \text { Castro } \quad \& \\
\text { Coutinho, } 2016\end{array}$ & $\begin{array}{l}\text { Assistência de enfermagem na oncologia } \\
\text { pediátrica }\end{array}$ & $\begin{array}{l}\text { Identificar quais as ações de enfermagem } \\
\text { prestadas à criança com câncer em tratamento } \\
\text { hospitalar. }\end{array}$ & $\begin{array}{l}\text { Revisão integrativa da } \\
\text { literatura }\end{array}$ \\
\hline Souza et al., 2017 & $\begin{array}{l}\text { Assistência de enfermagem ao indivíduo } \\
\text { idoso com múltiplas patologias } \\
\text { institucionalizado em ambiente hospitalar }\end{array}$ & $\begin{array}{l}\text { descrever o quadro clínico e a SAE de/para } \\
\text { uma paciente idosa portadora de Câncer (CA) } \\
\text { de colo uterino e CA de bexiga, Hipertensão } \\
\text { Arterial Sistêmica (HAS), Diabetes Mellitus } \\
\text { tipo } 2 \text { (DM II) e Herpez Zoster. }\end{array}$ & Relato de Experiência \\
\hline Borges et al., 2018 & $\begin{array}{l}\text { Avaliação dos Registros de Enfermagem } \\
\text { Acerca das Manifestações Clínicas de } \\
\text { Cardiotoxicidade }\end{array}$ & $\begin{array}{l}\text { Avaliar os registros de enfermagem em um } \\
\text { ambulatório de quimioterapia, acerca das } \\
\text { manifestações clínicas de cardiotoxicidade e } \\
\text { apresentar uma proposta de roteiro de } \\
\text { informações direcionada aos registros dos } \\
\text { cuidados nessa clientela. }\end{array}$ & $\begin{array}{l}\text { Estudo documental, } \\
\text { retrospectivo por análise } \\
\text { do prontuário e avaliação } \\
\text { quantitativa }\end{array}$ \\
\hline Pedrão et al., 2018 & $\begin{array}{l}\text { Diagnósticos e intervenções de } \\
\text { enfermagem para pacientes cardiológicos } \\
\text { em cuidados paliativos. }\end{array}$ & $\begin{array}{l}\text { Caracterizar o perfil clínico e } \\
\text { sociodemográfico e identificar os principais } \\
\text { diagnósticos e intervenções de Enfermagem. }\end{array}$ & Estudo quantitativo. \\
\hline Silva \& Moreira, 2018 & $\begin{array}{l}\text { Grau de complexidade dos cuidados de } \\
\text { enfermagem: }\end{array}$ & $\begin{array}{l}\text { Avaliar o grau de complexidade dos cuidados } \\
\text { de enfermagem de pacientes com câncer de }\end{array}$ & Estudo transversal. \\
\hline
\end{tabular}




\begin{tabular}{|c|c|c|c|}
\hline & $\begin{array}{l}\text { readmissões hospitalares de pessoas com } \\
\text { câncer de mama. }\end{array}$ & mama readmitidas na oncologia clínica. & \\
\hline Silva at el., 2018 & $\begin{array}{l}\text { Teorias de enfermagem aplicadas no } \\
\text { cuidado a pacientes oncológicos: } \\
\text { contribuição para prática clínica do } \\
\text { enfermeiro. }\end{array}$ & $\begin{array}{l}\text { Estudo é identificar as teorias de enfermagem } \\
\text { empregadas no cuidado a pacientes } \\
\text { oncológicos. }\end{array}$ & Revisão integrativa. \\
\hline Silva et al., 2019 & $\begin{array}{l}\text { Cuidados de enfermagem a pacientes } \\
\text { oncológicos: revisão integrativa }\end{array}$ & $\begin{array}{l}\text { Identificar os cuidados de enfermagem à } \\
\text { pacientes oncológicos. }\end{array}$ & Revisão integrativa. \\
\hline Rolim et al., 2019 & $\begin{array}{l}\text { Produção científica de enfermeiros } \\
\text { brasileiros sobre enfermagem e } \\
\text { oncologia: revisão narrativa da literatura. }\end{array}$ & $\begin{array}{l}\text { Conhecer o que tem sido produzido por } \\
\text { enfermeiros brasileiros sobre enfermagem e } \\
\text { oncologia. }\end{array}$ & Revisão narrativa. \\
\hline Campos et al., 2020 & $\begin{array}{l}\text { Impacto da fadiga na qualidade de vida } \\
\text { de mulheres com câncer de mama. }\end{array}$ & $\begin{array}{l}\text { Avaliar a qualidade de vida de mulheres com } \\
\text { fadiga secundária à quimioterapia durante o } \\
\text { tratamento para o câncer de mama. }\end{array}$ & $\begin{array}{l}\text { Estudo prospectivo, } \\
\text { quantitativo }\end{array}$ \\
\hline Silva et al,. 2020 & $\begin{array}{l}\text { Apoio social e qualidade de vida de } \\
\text { famílias de crianças com cardiopatia } \\
\text { congênita. }\end{array}$ & $\begin{array}{l}\text { Avaliar em que medida o apoio social a } \\
\text { famílias de crianças com cardiopatia congênita } \\
\text { impacta na qualidade de vida }\end{array}$ & Estudo Transversal \\
\hline Celeste \& Maia, 2021 & $\begin{array}{l}\text { Cuidados de enfermagem relacionados à } \\
\text { cardiotoxicidade envolvendo drogas } \\
\text { como a antraciclinas e anticorpo } \\
\text { monoclonais no tratamento oncológico }\end{array}$ & $\begin{array}{l}\text { Avaliar os cuidados de enfermagem no uso de } \\
\text { antineoplásicos tendo como efeito adverso } \\
\text { principal a cardiotoxicidade. }\end{array}$ & $\begin{array}{l}\text { Revisão bibliográfica } \\
\text { integrativa. }\end{array}$ \\
\hline
\end{tabular}

Fonte: Autores (2021).

\section{Discussão}

\section{Categoria A - Particularidades do tratamento contra o câncer em pacientes cardiopatas}

Atualmente, os pacientes cardiopatas em tratamento para câncer têm apresentado bastante sucesso, principalmente devido ao diagnóstico precoce e a utilização da quimioterapia adequada com auxílio da radioterapia que ajudou bastante no prognostico do câncer. Entretanto, os efeitos colaterais do tratamento, especialmente sobre o sistema cardiovascular têm aumentado o índice de morbidade mesmo após a cura da doença. Para isto não acontecer, o tratamento deve adotar estratégias para preservar o miocárdio, e a prevenção por meio de monitoramento efetivo é extremamente necessária para prevenir complicações (Santos \& Gallafrio, 2017).

Conforme Oliveira e Malachias (2019), uma das principais complicações está relacionada aos efeitos diretos do tratamento do câncer sobre a anatomia e fisiologia do coração e vasos sanguíneos, no qual pode promover uma isquemia miocárdica potencial ou enfatizar os fatores de risco associados ao uso de quimioterapia. Para oferecer um tratamento de qualidade e com segurança para o paciente, os profissionais de enfermagem devem sempre estar sempre atentos acerca dos sinais e sintomas de lesão cardíaca de decorrente do uso contínuo da quimioterapia antineoplásica.

O uso de agentes antineoplásicos no tratamento do câncer pode ocasionar certos efeitos secundários, tais como afecções cardiovasculares ou cardiotoxicidade, o que pode afetar a proposta terapêutica. A avaliação da função do coração de um paciente em uso de antineoplásicos deve ser contínua com o objetivo de reduzir os efeitos adversos e os problemas que atingem o sistema cardiovascular, como a alteração da função cardíaca, estrutura fisiológica e a bioquímica. Para obter melhor resposta no tratamento para ofertar qualidade na sobrevida e condições de vida adequada ao paciente (Mori, Bustamante \& Bustamante, 2016). 
O uso de antineoplásicos no tratamento do câncer, são fundamentais para controle oncológicos e hematológico, mas alguns quimioterápicos podem desencadear a alteração da função cardiovascular e ocasionar as cardiotoxicidade, alterações bioquímicas, inflamatórias que acometem o coração, o que leva a disfunção cardíaca e por fim, compromete a sobrevida do paciente (Costa et al., 2019).

Durante o tratamento do câncer é utilizado quimioterapia e antineoplásicos associados à radioterapia, que podem prejudicar muito o sistema cardiovascular dos pacientes que já possuem alguma doença cardíaca pré-existente. Dessa forma, é importante que ao planejar a terapêutica também sejam avaliados a função cardíaca, idade, marcadores bioquímicos, doses, intervalos e duração do tratamento, com o objetivo de minimizar possíveis danos que possam ser ocasionados pelos antineoplásicos (Abelin \& Renni, 2019).

Os quimioterápicos pertencentes à classe das antraciclinas e dos anticorpos monoclonais humanizados, como o trastuzumabe, produzem boa resposta no tratamento do câncer, porém existe risco de ocorrer cardiotoxicidade. Inúmeros parâmetros são vistos como preditores no aparecimento de toxicidade cardíaca, como a alteração contrátil segmentar ventricular esquerda (ACSVE), que é um preditor de cardiotoxicidade independente amplamente utilizado na detecção precoce da disfunção do músculo cardíaco e possui potencial para diminuir a morbidade e mortalidade em pacientes cardiopatas em tratamento contra o câncer (Barros et al., 2019).

As manifestações clínicas prejudiciais podem ocorrer durante o tratamento e por isso o tempo de utilização das medicações quimioterápicas e radioterápicas deve ser avaliado criteriosamente. A monitorização deve ser feita regularmente, especialmente relacionada à dosagem medicamentosa e ao intervalo entre os antineoplásicos associados à cardiotoxicidade, que podem levar o paciente a óbito durante o tratamento. Para prevenir complicações como essa, antes do início do tratamento deve ser realizada uma avaliação cardíaca para identificar e minimizar os danos no sistema cardíaco, ocasionados pelo uso da quimioterapia (Fonseca, 2017).

Durante o tratamento do câncer por meio de antineoplásico ocorre a perca ponderal de massa corpórea, o que irá afetar diretamente os sistemas do organismo e suas funções, com grande impacto em pacientes com problemas cardiovasculares, que apresentam diminuição da musculatura cardíaca e apresentam fadiga, falta de ar e desnutrição (Macêdo et al., 2020).

As manifestações clínicas da quimioterapia em pacientes cardiológicos podem ser descritas como: alterações bioquímicas, disfunção cárdica, falta de apetite, fadiga, náuseas, depressão, dor, sonolência, ansiedade, dispneia, mal-estar, estresse emocional, perda de massa muscular, anemia, aumento da atividade, inflamatória, alterações na coagulação e outros eventos, o que compromete a sobrevida do paciente e, consequentemente, altera a estratégia terapêutica de cardiovigilância e ocasiona a pausa ou a suspensão do tratamento (Borges et al., 2018).

Os pacientes com problemas cardiológicos ao serem submetidos ao tratamento por quimioterapia ou radioterapia podem apresentar complicações prematuras, morbidade e mortalidade por injúrias cardiovasculares isso faz com que o paciente necessite de uma abordagem multidisciplinar, que deve ser pautada em protocolos e diretrizes bem estabelecidas, com o intuito de desenvolver estratégias eficazes de prevenção e tratamento (Mesquita, 2016).

A atuação da equipe multiprofissional possui como um de seus objetivos, a prevenção de eventos adversos, alterações imunológicas, diminuição de agravos evitáveis, além da diminuição de riscos associados ao comprometimento ocasionados durante o tratamento. A doença cardiovascular e neoplasias compartilham muitos fatores de risco associados, com isso faz-se necessária a abordagem terapêutica associada para não comprometer o paciente (Acosta-Garduño \& Guillen-Rojas, 2017).

Hajjar et al. (2020) cita a necessidade da implementação de diretrizes para o uso de quimioterapia e radioterapia para o paciente oncológico com cardiopatia por meio de protocolos sistematizados, com o intuito de padronizar a prática clínica independentemente da cidade ou região da instituição de saúde. 
Os cuidados cardíacos durante o tratamento em uso de antineoplásicos sob terapêutica com alto grau de cardiotoxicidade visam otimizar a deteç̧ão precoce de cardiotoxicidade e podem utilizar os biomarcadores clínicos, laboratoriais, controle de ecocardiografia e de imagem, e estabelecer medidas intervencionistas para otimizar os cuidados cardiológicos, deve-se também avaliar a terapêutica oncológica para minimizar os riscos cardiovasculares à medida que as estratégias terapêuticas evoluem. Com o comprometimento cardiovascular durante a terapia oncológica, algumas modificações no tratamento podem ocorrer, que podem apresentar alteração na dosagem dos medicamentos, tempo de tratamento para cada droga, interrupção temporária ou definitiva da terapêutica, o que impede sua eficácia (Fiuza et al., 2016).

O diagnóstico precoce e tratamentos mais eficazes para o câncer proporcionam maiores possibilidades de cura e sobrevida a um importante número de pacientes. Principalmente por causa dos novos quimioterápicos, imunoterapia, modalidades de radioterapia mais eficazes e técnicas cirúrgicas mais avançadas. Por isso a necessidade de uma integração multidisciplinar das instituições de tratamento, ensino e pesquisa baseadas na prevenção, monitoramento ativo e intervenção terapêutica, contribuem para uma maior qualidade de vida aos pacientes (Santos, Siqueira, Renni, 2019).

\section{Categoria B - Cuidados de enfermagem no paciente com cardiopatia que está em tratamento de câncer com uso de quimioterapia e radioterapia}

O uso contínuo dos medicamentos antineoplásicos provoca efeitos adversos ligados ao sistema cardiovascular, como toxicidade hematológica, toxicidade gastrointestinal, cardiotoxicidade, hepatotoxicidade, toxicidade pulmonar, neurotoxicidade, problemas reprodutivos, toxicidade vesical e renal, alterações metabólicas, toxicidade dermatológica, reações alérgicas e anafilaxia e fadiga. Com o conhecimento acerca dos sinais e sintomas de toxidade do miocárdio, dos fatores agravantes e adequado registro dessas informações pela enfermagem, tornaram-se fundamentais para o manejo adequado e qualificado no tratamento do paciente. A enfermagem precisa estar atenta à visualização precoce do risco cardiovascular e do diagnóstico correto de alteração (Borges et al., 2018).

Os pacientes com comprometimento cardíaco submetidos à quimioterapia devem ter a função cardíaca avaliada e monitorada para estabelecer um modelo cardiovascular de base para comparação nas opções de tratamento. Essa avaliação deve incluir: preparo da história de doenças prévias e atuais e exame físico, desempenho do eletrocardiograma (ECG) e exames de imagem do coração. No plano de tratamento, além dos cuidados, é importante monitorar os sinais e sintomas de cardiotoxicidade, alterações clínicas, sinais vitais, antropometria, e ficar atento às complicações e efeitos colaterais durante o tratamento com os antineoplásicos. Portanto, o uso de quimioterápicos está associado a um potencial risco de cardiotoxicidade e é muito importante que durante a assistência de enfermagem ocorra a verificação do estado clínico do paciente durante todo o período de administração de medicamentos utilizados no combate ao câncer para avaliar qualquer evidência de alteração clínica. (Celeste \& Maia, 2021).

Os cuidados de enfermagem aos pacientes em tratamento de câncer com cardiopatia estão associados ao embasamento científico para obter melhor direcionamento na prática clínica. A teoria juntamente com a prática clínica fortalece o direcionamento de uma assistência de enfermagem segura e individualizada para cada paciente, por meio da Teoria humanística, autocuidado e transcultural, para poder transmitir isso a equipe de enfermagem com conhecimento e uma linguagem clara e objetiva para a sua qualidade de vida (Silva et al., 2018).

Antes de realizar os cuidados de enfermagem, o enfermeiro atua na tomada de decisão com a avaliação do grau de dependência do paciente para auxiliar na assistência de enfermagem, este tipo de classificação e capaz de direcionar e avaliar sua dependência, com isso auxilia a implementação e organização do cuidado de forma integral e com segurança (Silva \& Moreira, 2018). 
O enfermeiro deve realizar processo de enfermagem de forma humanizada e sistemática para que a assistência ocorra de maneira individualizada. O processo de enfermagem é classificado em 5 passos distintos: investigação, diagnóstico de enfermagem, planejamento, implementação ou intervenções de enfermagem e avaliação (Pedrão et al., 2018). Esse processo pode contribuir integralmente para um cuidado eficaz ao paciente oncológico com cardiopatia, por meio da sistematização da assistência de enfermagem (SAE) e possui um papel importante no acompanhamento de pacientes para abordar medidas educativas em saúde, prevenção de doenças e fragilidades, além de incentivar a autonomia e assegurar conforto e melhora no estilo de vida dos pacientes (Souza et al., 2017).

Silva et al. (2013) descreve que o enfermeiro deve identificar os principais diagnósticos e intervenções, avaliar as características, o perfil sociodemográfico e clínico do paciente. Os diagnósticos de enfermagem encontrados foram: intolerância à atividade, atividade física prejudicada, fadiga, déficit do autocuidado, desobstrução ineficaz das vias aéreas, padrão respiratório ineficaz, troca de gases prejudicada, risco de troca de gases prejudicada, perfusão tissular ineficaz, nutrição desequilibrada, integridade da pele prejudicada, dentição prejudicada, mucosa oral prejudicada, volume de líquidos excessivo, risco de infecção, risco de trauma, proteção ineficaz, constipação, incontinência urinária, dor, confusão aguda, ansiedade, medo, padrão de sono perturbado, comunicação verbal prejudicada e enfrentamento ineficaz.

O enfermeiro, ao realizar a assistência aos pacientes oncológicos com cardiopatia, também deve direcionar seus cuidados junto à família, para que consiga desenvolver uma forma educativa que irá contribuir positivamente para a recuperação do paciente e atrasar a progressão da doença. A equipe de enfermagem deve estar presente para tentar auxiliar da melhor forma as necessidades do paciente, seus sentimentos e angústias mostradas durante todo o tratamento (Rolim et al., 2019).

Para Campos et al. (2020) o cuidado de enfermagem a pacientes oncológicos com cardiopatia inclui: educação para o autocuidado, soroterapia, preparo do cólon, gerenciamento de medicamentos, posicionamento do paciente, monitoramento de sinais vitais, cuidados com tubo de infusão, controle da ingesta hídrica, mudanças de decúbito de acordo com a necessidade previamente diagnosticada pelo enfermeiro, registros médicos, de enfermagem e referências de exames, curativo diário, garantia da permeabilidade das vias aéreas e controle da dor (Silva et al., 2019).

\section{Considerações Finais}

Os pacientes em tratamento contra o câncer que também são acometidos por alguma cardiopatia devem ser seu risco de comprometimento cardíaco avaliado antes e após a utilização de quimioterápicos, com o objetivo de investigar uma possível cardiotoxidade, relacionada a administração de tais medicamentos. A avaliação da função cardíaca deve ser realizada de maneira constante para verificar uma possível lesão cardíaca e/ou o acometimento de vasos.

Durante o uso de quimioterapia, é possível que o paciente desenvolva uma resposta inflamatória e ocorra manifestações clínicas que podem ser agravadas em pacientes cardiopatas, fato que está relacionado principalmente a cardiotoxicidade por quimioterápicos. Durante o tratamento é possível que ocorra perda da massa corporal, além de alterações bioquímicas e mais especificamente, na coagulação sanguínea. É importante que se realize a detecção precoce de cardiotoxicidade, por meio da utilização de biomarcadores clínicos, laboratoriais, controle de ecocardiografia e de imagem, além disso, cabe ao profissional responsável pelo paciente, estabelecer estratégias de intervenção para otimizar os cuidados cardiológicos. Devido a essas particularidades com esse tipo de paciente, é necessário que sejam elaborados e implantadas diretrizes para o uso de quimioterapia e radioterapia, pois somente com uma prática clínica padronizada e sistematizada, será possível garantir a segurança no tratamento do paciente oncológico com cardiopatia.

Os principais cuidados de enfermagem em pacientes oncológicos com cardiopatia são: intervenções de educação de 
autocuidado, monitoramento de sinais vitais, mudanças de decúbito de acordo com a necessidade previamente diagnosticada pelo enfermeiro, posicionamento do paciente, gerenciamento de medicamentos, programas de tratamento como: soroterapia, preparo do cólon, cuidados com tubo de infusão, registros médicos, de enfermagem e referências de exames, controle da ingesta hídrica, garantia da permeabilidade das vias aéreas, curativo diário e controle da dor. Espera-se que este trabalho contribua para melhorar a sistematização da assistência ao paciente oncológico cardiopata, assim como contribuir com publicações futuras a respeito dessa temática.

\section{Referências}

Abelin, T. \& Renni, M. J. P. (2019). Insuficiência Cardíaca no Paciente Oncológico: Preditores de Risco. Revista Brasileira de Cancerologia. 65(3): e-13719.

Acosta-Garduño. F., \& Guillen-Rojas, R. (2017) Riesgo cardiovascular en cirugía oncológica. Revista mexicana de Anestesiologia. 40(1).

Barros, M. V. L. et al. (2019). Alteração Contrátil Segmentar Ventricular Esquerda é Preditor Independente de Cardiotoxicidade em Pacientes com Câncer de Mama em Tratamento Quimioterápico. Arquivos Brasileiros de Cardiologia. 112(1). 50-56.

Borges, J. A. et al. (2018) Fadiga: Um Sintoma Complexo e seu Impacto no Câncer e na Insuficiência Cardíaca. International Journal of Cardiovascular Sciences. 31(4). 433-442.

Borges, M. D et al. (2018). Avaliação dos Registros de Enfermagem Acerca das Manifestações Clínicas de Cardiotoxicidade. Revista Enfermagem Atual In Derme. 86(24).

Campos, C. S et al. (2020). Impacto da fadiga na qualidade de vida de mulheres com câncer de mama. Revista Família, Ciclos de Vida e Saúde no Contexto Social. 8 (3). 383-391.

Celeste, L. E. N. \& Maia, M. R. (2021) Cuidados de enfermagem relacionados à cardiotoxicidade envolvendo drogas como a antraciclinas e anticorpo monoclonais no tratamento oncológico. Revista PubSaúde. 5(905).

Cesário, J. M. S., Flauzino, V. H. P. \& Mejia, J. V. C. (2020). Metodologia científica: Principais tipos de pesquisas e suas caraterísticas. Revista Científica Multidisciplinar Núcleo do Conhecimento, 5(11). 23-33. 10.32749/nucleodoconhecimento.com.br/educacao/tipos-de-pesquisas

Costa, K. C et al. (2019). Quimioterápico promove inflamação no tecido cardíaco. XI Encontro internacional de produção cientifica.

Fiuza, M. et al. (2016). Organização e implementação de uma consulta de cardio-oncologia. Revista Portuguesa de Cardiologia. 35(9). 485-494.

Fonseca, C. M. R. A. C. S. (2017). O tratamento oncológico e a doença arterial coronariana. Sociedade de Cardiologia do Estado de São Paulo. 27(4). 294301.

Hajjar, L. A et al. (2020). Diretriz Brasileira de Cardio-oncologia - 2020. Arquivo. Brasileiro de Cardiologia. 115(5). 1006-1043.

Macêdo, P. F. C et al. (2020.) Fatores associados ao excesso de adiposidade em pacientes com câncer de mama sob tratamento quimioterápico em um hospital oncológico de referência em Pernambuco - Brasil. Brazilian Journal of Development. 6 (4). 21871 - 21884.

Mesquita, C. T. (2016). O Surgimento da Cardio-Oncologia. International Journal of Cardiovascular Sciences. 29(6). 419-421.

Mori, E. R., Bustamante, L. A., Bustamante, J.B. (2016). Insuficiencia cardíaca por quimioterapia. Revista Sociedade Peruana de Medicina Interna. 29(2). $15-25$.

Oliveira, G. M. M., \& Malachias, M. V. B. (2019). Novos horizontes da Cardio-Oncologia. Revista Brasileira de Cancerologia. 65(3). e-05585.

Pedrão, T. G. G et al. (2018). Diagnósticos e intervenções de enfermagem para pacientes cardiológicos em cuidados paliativos. Revista enfermagem Universidade Federal de Pernambuco. 12(11). 3038-45.

Peters, M. D et al. (2015). Guidance for conducting systematic scoping reviews. International Journal of Evidence-Based Healthcare. 13(3). 141-146. 10.1097/XEB.0000000000000050.

Pinna Junior, B. J. B. et al. (2020). O uso de dispositivos cardíacos eletrônicos implantáveis durante tratamento oncológico: uma revisão. Revista Norte Nordeste de Cardiologia. 10(3) 6-9.

Rolim, D. S et al. (2019). Produção científica de enfermeiros brasileiros sobre enfermagem e oncologia: revisão narrativa da literatura. Arquivos de ciências da saúde da Universidade Paranaense. 23(1). 41-47.

Santos, M. V. C., Siqueira, A. S. \& Renni, M. J. P. (2019). Cardio-Oncologia no Brasil: Cenário Atual e Perspectivas. Revista Brasileira de Cancerologia. 65(3). 15-23

Santos, M. V. C. \& Gallafrio, C. C. (2017) Cardio-oncologia na população pediátrica. Revista Sociedade de Cardiologia do Estado de São Paulo. 27(4). 2829.

Silva, G. V et al. (2020). Apoio social e qualidade de vida de famílias de crianças com cardiopatia congênita. Ciência \& Saúde Coletiva, 25(1). 3153-3162. 
Research, Society and Development, v. 10, n. 6, e34310615357, 2021

(CC BY 4.0) | ISSN 2525-3409 | DOI: http://dx.doi.org/10.33448/rsd-v10i6.15357

Silva, F. S et al. (2019). Cuidados de enfermagem a pacientes oncológicos: revisão integrativa. Revista Research Society and Development. 8(6). e35861037. $10.33448 /$ rsd-v8i6.1037.

Silva, T. P. (2013). Cuidados de enfermagem à criança com câncer: uma revisão integrativa da literatura. Revista de enfermagem da UFSM, 1(1). 68-78. $10.5902 / 217976926918$

Silva, L. G. \& Moreira, M. C. (2018). Grau de complexidade dos cuidados de enfermagem: Readmissões hospitalares de pessoas com câncer de mama. Revista Gaúcha Enfermagem. 1(39), e20180015. https://doi.org/10.1590/1983-1447.2018.20180015.

Silva, N. R. F et al. (2018) Teorias de enfermagem aplicadas no cuidado a pacientes oncológicos: contribuição para prática clínica do enfermeiro. Revista Uningá. Maringá, 55(2). 59-71.

Souza, F. B. T. L et al. (2017). Assistência de enfermagem ao indivíduo idoso com múltiplas patologias institucionalizado em ambiente hospitalar. Anais V. Congresso internacional de envelhecimento humano.

Vieira, A. P. M. S., Castro, D. L., \& Coutinho, M. S. (2016). Assistência de enfermagem na oncologia pediátrica. Revista Eletrônica Atual Saúde, 3(3). 67-75. 